\section{Traduzindo a pandemia da COVID- 19: um relato de experiência}

\author{
Translating COVID-19 pandemic: an experience \\ report
}

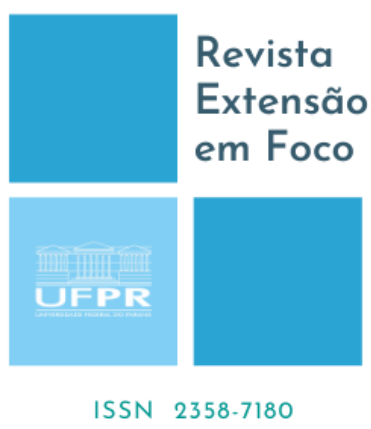

\author{
Anna Luiza Maffessoni ${ }^{1}$, Amanda Nardi Zanluchi ${ }^{2}$, Bianca Bianca Melo de Assis ${ }^{3}$, \\ Bianca Rojo $^{4}$, Isadora Maria Pilati Campos ${ }^{5}$, Pablo Guarisco Ferreira ${ }^{6}$, Victoria \\ Castello Branco Iwakami de Mattos ${ }^{7}$, Kleber Fernando Pereira ${ }^{8}$, Patrícia Leen Kosako \\ Cerutti $^{9}$, Rafael Lírio Bortoncello ${ }^{10}$, Eduardo Gomes de Souza ${ }^{11}$, Ana Paula Carneiro \\ Brandalize $^{12}$
}

\begin{abstract}
RESUMO
Objetivo: Relatar a experiência de alunos e professores de Medicina na participação de um projeto de tradução de artigos científicos sobre o novo coronavírus para a língua portuguesa, a fim de democratizar o acesso deste conteúdo a profissionais de saúde e à população geral. Metodologia: 35 discentes e 5 docentes da Universidade Federal do Paraná Campus Toledo se organizaram remotamente em grupos de WhatsApp para traduzir artigos científicos publicados em inglês sobre a COVID-19. Os professores selecionaram artigos relevantes de bases de dados como Pubmed. Os artigos traduzidos foram publicados no website da universidade e artes gráficas com os destaques de cada um, nas redes sociais. Resultados e discussão: Em meio à pandemia da COVID-19 e seus desafios, docentes e discentes do Curso de Medicina viram na tradução de artigos científicos sobre o novo coronavírus uma oportunidade de seguir contribuindo com a sociedade por meio de uma ação de caráter extensionista. Foram traduzidos 69 artigos em sua integralidade. Desta maneira, o projeto permitiu a propagação de conhecimento confiável em meio à atual epidemia das fake news, bem como oportunizar aos discentes tivessem o contato direto com artigos científicos publicados em língua estrangeira, que traziam linguajar médico-científico. Conclusão: O projeto mostrou-se uma boa estratégia de manutenção de atividades extensionistas no contexto da pandemia, possibilitando maior acesso às informações aos profissionais de saúde e à população em geral. Aos participantes, permitiu aprendizado acerca de leitura de artigos científicos e linguagem técnica na língua inglesa.
\end{abstract}

Palavras-chave: COVID-19; Tradução de artigos; Extensão.

\title{
ABSTRACT
}

Objective: Report Professors and medical students experience as part of a project to translate to Portuguese scientific articles about the new coronavirus, in order to democratize the access to this content among healthcare professionals and the general population. Methods: 35 students and 5 Professors of Federal University of Parana - Campus Toledo gathered online through WhatsApp groups, in order to translate scientific articles published in english about COVID-19. Professors selected relevant articles in databases such as Pubmed. The translated articles were published in the University's website, and graphic arts with the highlights of each article were published in social media. Results and discussion: In times of COVID-19 pandemic and its challenges, Professors and students of the Medical School saw an opportunity to contribute with society through an extensionist action of translating scientific articles. A total of 69 articles were fully translated. Thus, the project allowed the spread of trustworthy information amid the current fake news "infodemics", and also offered the students the opportunity to be in contact with scientific articles published in english and with technical medicalscientific language. Conclusion: The project turned out to be a good strategy of maintaining extensionist activities during the pandemic, allowing healthcare professionals and the general population a broader access to information. All participants were able to learn more about reading scientific articles and also about technical writing in English. 


\footnotetext{
${ }^{1}$ Graduanda em Medicina. Universidade Federal do Paraná (UFPR), Toledo, Paraná, Brasil. E-mail: anna.maffessoni@gmail.com. Orcid: https://orcid.org/0000-0002-5826-1778

${ }^{2}$ Graduanda em Medicina. Universidade Federal do Paraná (UFPR), Toledo, Paraná, Brasil. Email: amandanzanluchi@gmail.com. Orcid: https://orcid.org/0000-0003-0083-7786

${ }^{3}$ Graduanda em Medicina. Universidade Federal do Paraná (UFPR), Toledo, Paraná, Brasil. Email: biancaluiza@ufpr.br. Orcid: https://orcid.org/0000-0001-8163-2832

${ }^{4}$ Graduanda em Medicina. Universidade Federal do Paraná (UFPR), Toledo, Paraná, Brasil. Email: bianca@ rojo.com.br. Orcidhttps://orcid.org/0000-0002-5011-3834

${ }^{5}$ Graduanda em Medicina. Universidade Federal do Paraná (UFPR), Toledo, Paraná, Brasil. Email: isapilati@gmail.com. Orcid: https://orcid.org/0000-0001-5264-1836

${ }^{6}$ Graduando em Medicina. Universidade Federal do Paraná (UFPR), Toledo, Paraná, Brasil. Email: pabloguarisco@gmail.com. Orcid: https://orcid.org/0000-0002-8842-6190

${ }^{7}$ Graduanda em Medicina. Universidade Federal do Paraná (UFPR), Toledo, Paraná, Brasil. Email: victoriacbim.ufpr@gmail.com. Orcid: https://orcid.org/0000-0002-1101-7487

${ }^{8}$ Doutor em Anatomia Comparativa. Universidade Federal do Paraná (UFPR), Toledo, Paraná, Brasil. E-mail: kleber.ufpr@gmail.com. Orcid: https://orcid.org/0000-0002-5102-6273

${ }^{9}$ Médica Ginecologista e Obstetra. Universidade Federal do Paraná (UFPR), Toledo, Paraná, Brasil. E-mail: patriciakosako@ufpr.br. Orcid: https://orcid.org/0000-0003-3089-8029

${ }^{10}$ Médico Radiologista. Universidade Federal do Paraná (UFPR), Toledo, Paraná, Brasil. E-mail: rafaelbotoncello@hotmail.com. Orcid: https://orcid.org/0000-0002-9294-1136

${ }^{11}$ Médico Urologista. Universidade Federal do Paraná (UFPR), Toledo, Paraná, Brasil. E-mail: prof.eduardo@ufpr.br. Orcid: https://orcid.org/0000-0002-3979-4974

${ }^{1}$ Doutora em Genética e Biologia Molecular. Universidade Federal do Paraná (UFPR), Toledo, Paraná, Brasil. E-mail: anapaulabrandalize@yahoo.com.br. Orcid: https://orcid.org/0000-0002-5293-8186
} 


\section{INTRODUÇÃO}

No final de 2019, um surto de pneumonia de causa desconhecida na cidade de Wuhan, na China, colocou as autoridades de saúde locais em alerta (Zhu et al., 2019). Inicialmente associado à exposição dos pacientes em um mercado de frutos do mar da cidade, o surto teve sua causa definitiva identificada em 31 de dezembro de 2019: um novo tipo de coronavírus (OMS, 2020). O SARS-CoV-2, assim nomeado posteriormente, apesar de desconhecido, era alarmante. Cerca de um mês após sua identificação, o vírus já estava presente em 18 países fora da China e, em 11 de março de 2020, a COVID-19 (doença do coronavírus 2019) já era considerada uma pandemia pela Organização Mundial da Saúde (OMS, 2020).

Desde então, órgãos de saúde do mundo todo vêm unindo esforços para, através da análise de resultados de pesquisa básica, ensaios clínicos e estudos epidemiológicos, entregar informações acerca do novo coronavírus. Tais ações têm a importante missão de orientar a aplicação de protocolos de tratamento, prevenção e controle da pandemia da COVID-19. No entanto, a grande maioria das informações é disponibilizada através de artigos científicos publicados em língua inglesa. Tal fator limita o acesso ao conteúdo a uma pequena parcela da população, especialmente no Brasil, onde apenas 5,1\% da população com 16 anos ou mais afirma possuir algum conhecimento no idioma (DATA POPULAR, 2013). Ao mesmo tempo, a divulgação de artigos na língua inglesa representa uma barreira à transposição da ciência em nosso país, restringindo o acesso a quem poderia usufruí-la.

Com o grande volume de informações disponibilizadas atualmente sobre a pandemia da COVID-19, a humanidade depara-se com outro desafio: a infodemia. Segundo a OMS, tal termo refere-se a um "excesso de informações, algumas precisas e outras não, que tornam difícil encontrar fontes idôneas e orientações confiáveis quando se precisa”. Essa problemática, entre outras consequências, acaba por dificultar a resposta às emergências de saúde, uma vez que prejudica o acesso a fontes confiáveis de informação pela população de modo geral, bem como pelos responsáveis por decisões imediatas em um atendimento em saúde (ORGANIZAÇÃO PAN-AMERICANA DE SAÚDE, 2020). 
Em meio a esse cenário, informações que carecem de valor científico, popularmente conhecidas como Fake News, ganham espaço nas mídias sociais. Sem acesso à informação de qualidade, o grande público que utiliza as redes sociais como único meio de atualização sobre a atual pandemia é incapaz de discernir entre o conhecimento científico e o senso comum. Além disso, a disseminação de desinformações traz repercussões graves, que comprometem diretamente a saúde pública (Europol, 2020). Exemplo dessa repercussão foram as notícias liberadas precocemente acerca do possível efeito benéfico da hidroxicloroquina no tratamento e cura da COVID19, o que fez com que os estoques do antimalárico chegassem ao esgotamento em farmácias, em poucos dias, situação que atingiu diretamente os pacientes em uso do medicamento para tratamento de outras condições clínicas (Folha de S. Paulo, 2020).

Em uma era onde as fake news confrontam constantemente a prática da Medicina Baseada em Evidências, a leitura de artigos científicos torna-se essencial aos profissionais de saúde. Estes devem sempre buscar evidências de eficácia, efetividade e segurança para nortear decisões clínicas e aumentar as chances de acerto em diagnósticos e condutas (ATTALAH, 2018).

Dentro do contexto da problemática e do impacto da pandemia da COVID-19, 35 alunos do curso de Medicina da Universidade Federal do Paraná (UFPR), Campus Toledo, juntamente com professores orientadores, reuniram-se em um Programa de Voluntariado Acadêmico (PVA) para a tradução de artigos internacionais, recentes e relevantes, sobre o novo coronavírus. O principal objetivo do projeto de tradução de artigos sobre o novo coronavírus foi disponibilizar, de forma organizada e gratuita, informações confiáveis e atualizadas sobre a COVID-19 em língua portuguesa, especialmente aos profissionais de saúde, mas também à população geral.

\section{MÉTODOS}

Este artigo apresenta um relato de experiência do projeto de voluntariado acadêmico, com caráter extensionista, intitulado "Tradução livre de artigos científicos sobre a COVID-19”. As atividades foram realizadas com a participação de 35 alunos do 
curso de Medicina da UFPR Campus Toledo, matriculados do $2^{\circ}$ ao $9^{\circ}$ período do curso, além de cinco professores, também vinculados à universidade.

Para iniciar o projeto, os participantes foram organizados em cinco grupos, sendo cada grupo composto por um docente e cerca de seis a oito alunos. A realização das traduções foi feita integralmente de forma remota, sendo a comunicação entre os integrantes de cada grupo, e entre grupos, realizada por meio do aplicativo WhatsApp.

As atividades foram desenvolvidas no período de março a maio do ano de 2020 e estruturadas em cinco etapas, que ocorriam simultaneamente entre os diferentes grupos criados:

1- Seleção de artigos: os professores coordenadores de cada grupo selecionaram artigos científicos recentes e publicados em língua inglesa sobre a COVID-19 encontrados em bases de dados científicos como PubMed, Scopus, Scielo e UpToDate. A relevância do conteúdo de artigos aplicados a área da saúde era então discutida entre professores e discentes, para então serem selecionados para tradução.

2- Tradução do artigo científico previamente selecionado: dentro de cada grupo, uma dupla de alunos ficou responsável por traduzir os artigos sobre a COVID-19 da língua inglesa para a portuguesa. Todos os artigos traduzidos foram inseridos em um template próprio, o qual continha informações sobre os autores da tradução.

3- Revisão do artigo traduzido: outra dupla de alunos revisou o artigo traduzido por seus colegas;

4- Elaboração de material informativo para divulgação em redes sociais: uma dupla de alunos elaborou um resumo dos principais achados científicos de cada artigo selecionado. Tais achados foram posteriormente inseridos em uma arte gráfica e publicados sob forma de highlights (destaques) nas redes sociais Facebook e Instagram da UFPR Campus Toledo e Centro Acadêmico de Medicina de Toledo (CAMTO UFPR).

5- Revisão final e publicação: cada professor coordenador de grupo revisou todos os artigos traduzidos e enviou feedbacks acerca da atividade desenvolvida. Logo após, o artigo foi publicado em sua integralidade no site da universidade (http://www.toledo.ufpr.br/portal/artigos-cientificos-covid-19/). 


\section{RESULTADOS E DISCUSSÃO}

A Extensão Universitária é um processo educativo, cultural, científico e/ou tecnológico que compõe um dos três pilares da Universidade - ensino, pesquisa e extensão. A extensão articula o ensino e a pesquisa de forma indissociável e viabiliza a relação transformadora entre a Universidade e os demais setores da sociedade (Resolução 72/11 - CEPE Art. 1). As atividades extensionistas objetivam suprir os interesses da própria Instituição de Ensino Superior ao corroborar com a aprendizagem do corpo discente, além de trazer benefícios diversos à sociedade a qual a instituição está inserida (SILVA ALB, et al, 2019). Apesar de a tradução de artigos sobre o novo coronavírus estar oficializada como um Programa de Voluntariado Acadêmico (PVA), o trabalho possui caráter extensionista por objetivar trazer benefícios aos próprios integrantes da atividade e à toda a sociedade que tem sido afetada pela pandemia (NUNES et al., 2011).

Assim, inseridos em um novo contexto de pandemia da COVID-19, docentes e discentes do curso de Medicina da Universidade Federal do Paraná - Campus Toledo, foram impelidos a descobrir formas de contribuir positivamente com a sociedade. Em meio a interrupção das atividades de ensino presenciais em março do ano de 2020, 35 alunos, orientados por cinco professores, se reuniram com o objetivo de traduzir artigos científicos publicados em inglês sobre a COVID-19. Durante a vigência do projeto foram traduzidos 64 artigos os quais trouxeram informações relevantes publicadas nos principais periódicos internacionais sobre a evolução mundial da pandemia. O foco de tais artigos abrangia a prevenção da infecção, exames clínicos e laboratoriais, novos tratamentos, formas de infecção e apresentações clínicas da doença COVID-19. As traduções foram publicadas em template personalizado na página do curso de Medicina da Universidade Federal do Paraná, Campus Toledo (Figura 1). 
Figura 1 - Layout de um artigo científico traduzido publicado no site da UFPR Campus Toledo

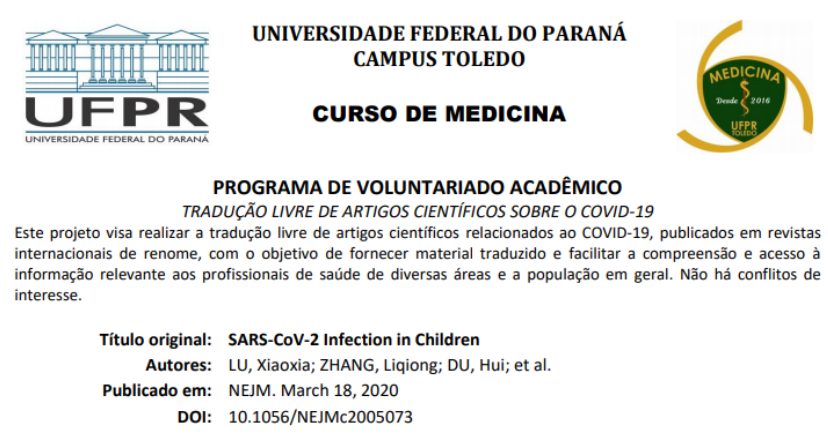

Fonte:

Campus Toledo (http://www.toledo.ufpr.br/portal/wp-

Website UFPR content/uploads/2020/03/Infec\%C3\%A7\%C3\%A3o-por-SARS-CoV-2-em-Crian\%C3\%A7as.pdf)

Diferentemente dos demais projetos de caráter extensionista já realizados neste Campus, o projeto aqui descrito contemplou dificuldades inéditas a qualquer universidade: o isolamento social do início da pandemia. O desenvolvimento de projetos de extensão à distância tornou-se um desafio, já que a extensão visa o contato e envolvimento com a sociedade. Por meio de atividades extensionistas, é possível inserir o estudante na realidade diária e nos contextos políticos de determinado local a fim de estreitar laços entre o meio científico e a sociedade (FUNAI; SILVA; ZANETTINI; HANAUER; SILVA; VALCARENGHI; CANCI; KLAUS, 2020). No entanto, diante do cenário de lockdown imposto pela pandemia, o diálogo entre universidade e sociedade precisou ser adaptado para um formato que não oferecesse riscos aos acadêmicos e à população. Para suprir esse revés e fazer com que a universidade cumpra sua função social mesmo em meio à situação de uma pandemia, a execução do projeto se deu por completo através de plataformas digitais. Em um primeiro momento, a utilização do aplicativo Whatsapp foi fundamental para a execução das atividades à distância. Os grupos formados foram beneficiados pela comunicação rápida e eficaz troca de informações sobre a seleção dos artigos, bem como pela discussão dos tópicos mais relevantes acerca da evolução e impactos da COVID-19.

O presente projeto de tradução de artigos se enquadra na categoria de plataformas de tradução de conhecimento. El-Jardali, Bou-Karroum e Fadlallah (2020) denominaram tais plataformas de acesso à informação científica traduzida "plataformas de tradução de conhecimento" (knowledge translation platforms). O papel dessas plataformas é permitir que, através da disponibilidade de artigos na língua materna, em um compilado 
organizado e acessível, seja facilitado o diálogo em saúde, a tomada de decisões informadas e até a determinação de políticas de saúde pública. Através do acesso ao website da UFPR Campus Toledo, os profissionais de saúde puderam ler artigos científicos internacionais em datas próximas às publicações originais, já disponibilizados em língua portuguesa. A tarefa de classificar informações em confiáveis ou não se tornou menos dispendiosa, o que permitiu aos profissionais a aplicação de conhecimento teórico de forma prática, ao diminuir a distância entre a ciência, política e rápida tomada de decisões (EL-JARDALI; BOU-KARROUM; FADLALLAH, 2020). Frente a essa situação, outras entidades também se organizaram para distribuir informações confiáveis de maneira organizada.

No entanto, uma grande barreira para esta prática ainda é o idioma: o inglês é a língua dominante no meio científico (MENEGHINI; PACKER, 2007). Isso é ainda mais relevante em países em desenvolvimento, como o Brasil, no qual apenas 5,1\% da população declara ter algum conhecimento da língua inglesa, sendo que destes, apenas 16\% considera seu nível de inglês avançado ou fluente (British Council, 2013). A tradução de qualquer obra vai muito além do que meramente trazer o significado isolado da palavra original para o idioma em que deve ser compreendida. As relações léxicas entre uma língua e outra podem ser variáveis e palavras podem apresentar significados distintos em diferentes idiomas, bem como podem não apresentar um equivalente na língua para qual será traduzida (OLIVEIRA, 2017). Mais do que trazer os dados técnicos apresentados nos originais, o processo de tradução visou, sem perder a credibilidade e a veracidade das informações, articular de forma simplificada o conteúdo do artigo para que pudesse ser compreendido pelas pessoas que entrassem em contato com o material.

Os acadêmicos também precisaram desenvolver e aprimorar competências para que as etapas do projeto fossem realizadas. Conforme Serrão (2020), extensionistas têm sido desafiados a extrair de si mesmos novas habilidades talvez antes não imaginadas e/ou exigidas. Os acadêmicos por guardarem uma ligação mais recente com um mundo menos acostumado aos jargões científicos, a partir de intensa reflexão e estudo, tanto do conhecimento científico como da melhor forma de sua expressão em sua língua materna. Tal processo só pode ser desenhado a partir da expressão da língua traduzida que permite a compreensão pela comunidade dos dados contidos nos originais dos artigos traduzidos, Britto (2012) ressalta que Schleiermacher já realizava essa reflexão desde meados de 1700. O resultado dessa multiplicação de informações viabilizadas pelo projeto foi 
destaque em publicação veiculada pela revista PIAUÍ. Em entrevista concedida à revista, uma médica que atua na atenção básica de saúde na cidade de Boa Vista, em Roraima, descobriu a iniciativa por meio das redes sociais. Segundo ela, os artigos auxiliaram na atualização dos estudos em andamento em relação aos tratamentos, protocolos sobre a doença e cuidados no dia a dia para evitar a infecção pelo novo coronavírus (Figura 2). A mesma publicação ainda ressaltou que a divulgação do projeto nas redes sociais possibilitou que a população leiga tivesse acesso a informações confiáveis e com embasamento científico (LOPES, 2020).

Figura 2 - Print da Reportagem que destaca o projeto de tradução de artigos científicos da UFPR Campus Toledo

\section{piauí}

questōes cientificas

\section{TRADUZINDO A PANDEMIA}

Sem aulas na quarentena, professores $\theta$ estudantes universitários oriam núcleos de tradução para divulgar artigos científicos $\theta$ combater informações falsas sobre a Covid-19

PLÍNIO LOPES

19maio2020_17h43

Fonte: Revisa Piauí (https://www.facebook.com/UFPRoficial/posts/3890247737715379)

A extensão tornou-se, neste momento de pandemia, algo de grande relevância e, como afirma Diniz et al (2020), articular e realizar novos mecanismos de atuação na extensão é imperativo. Ainda, a prática extensionista torna-se necessária e oportuna por fazer a universidade se ressignificar diante da sociedade para além dos limites acadêmicos (BARBOSA, 2020). Por mais que já existam iniciativas para tornar o conhecimento científico mais acessível, as publicações científicas ainda são elitizadas e têm seu alcance restrito àqueles que compreendem a Língua Inglesa e os termos técnicos de determinada área. $\mathrm{O}$ atual sistema de comunicação científica não atende às necessidades da ciência $\mathrm{e}$ da sociedade (LARIVIÈRE; SHU; SUGIMOTO, 2020), isso reforça a importância de ações que visem mudar o curso desse cenário.

Com o intuito de difundir de maneira eficiente os principais conteúdos descritos nos artigos traduzidos, foram elaborados highlights de cada artigo para ampla divulgação via internet (Figura 3). Tal processo ampliou ainda mais o acesso às informações por 
meio de redes sociais. Segundo as métricas da rede social Instagram, desde o início do projeto até o dia 10 de abril do ano de 2021, foram aproximadamente 9.000 usuários únicos alcançados, cerca de 700 curtidas e 140 compartilhamentos. Ainda, o projeto foi divulgado nas redes oficiais da UFPR (Facebook e Twitter), o que possibilitou que alcançasse todos os Campi da Universidade (Figuras 4 e 5).

Figura 3 - Highlights de um dos artigos traduzidos pelo projeto

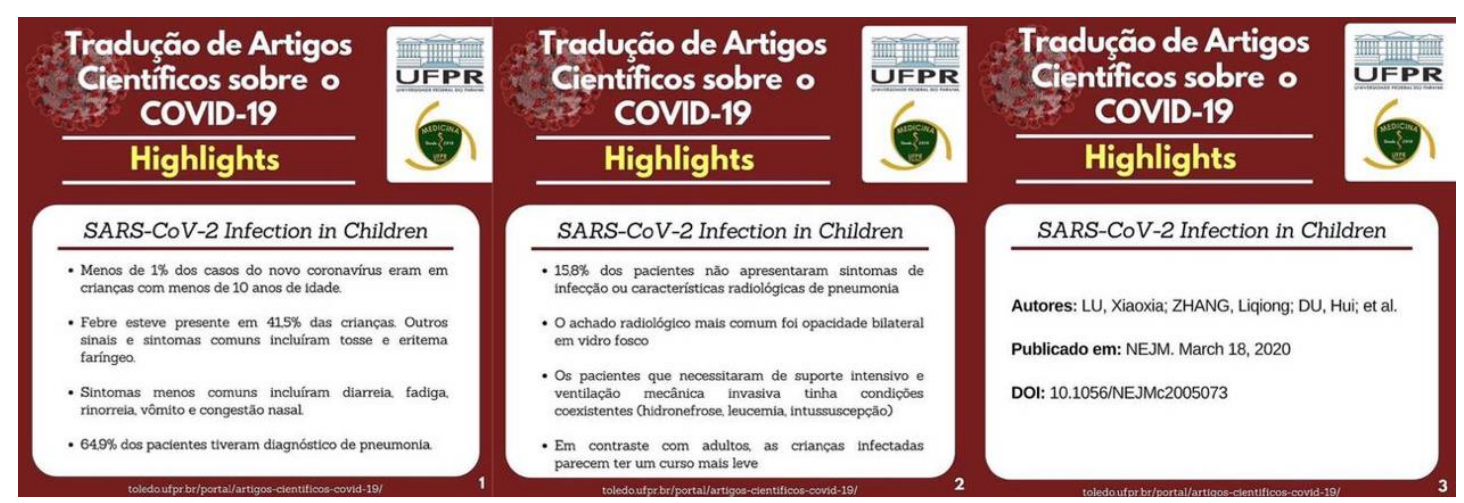

Fonte: Instagram (https://www.instagram.com/p/B-LSptaFS6u/?igshid=1md0grtgiexd8)

Figura 4 - Divulgação do projeto de tradução de artigos científicos no Facebook da UFPR

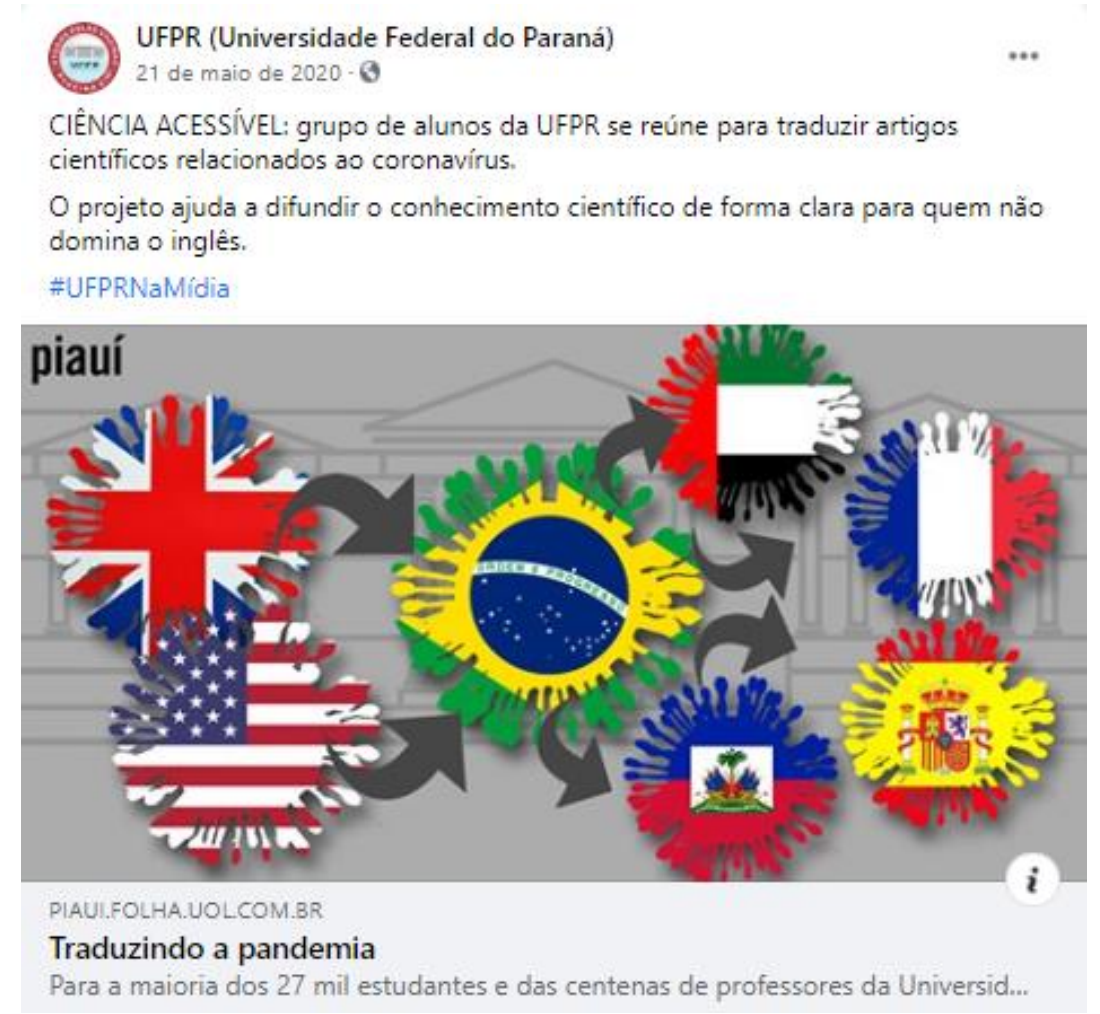

Fonte: Facebook (https://www.facebook.com/UFPRoficial/posts/3890247737715379) 
Figura 5 - Divulgação do projeto de tradução de artigos científicos no Twitter da UFPR

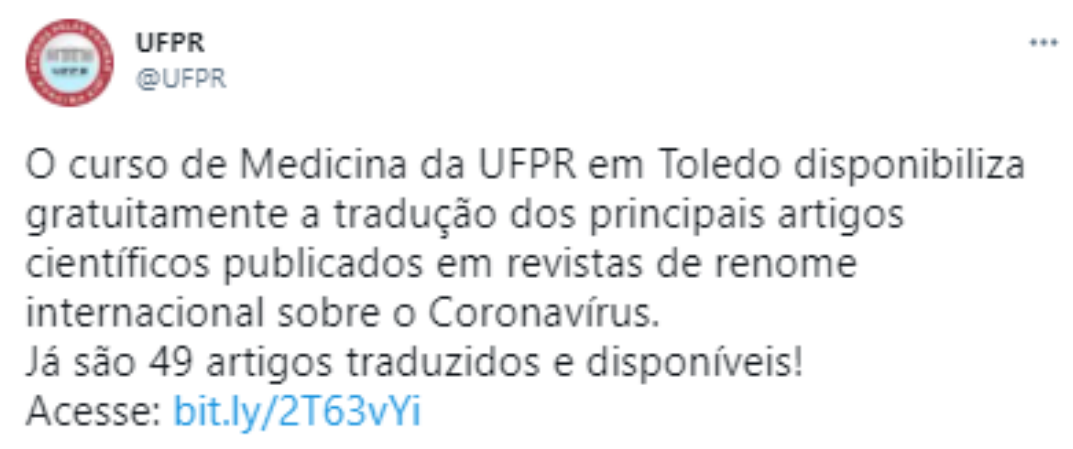

Fonte: Twitter (https://twitter.com/UFPR/status/1261351319186452480)

A disponibilização dos artigos científicos traduzidos sobre o novo coronavírus também permitiu a propagação de conhecimento confiável em meio à atual epidemia das fake news, democratizando o acesso à informação. Um estudo recente realizado em duas etapas por pesquisadoras da Escola Nacional de Saúde Pública Sergio Arouca (ENSP) analisou denúncias de notícias falsas sobre a COVID-19 recebidas pelo aplicativo "Eu fiscalizo". Na primeira etapa, a maior parte das notícias concentrou textos envolvendo métodos caseiros para curar ou prevenir a doença. Na segunda etapa, 24,6\% das notícias afirmava que a doença é uma estratégia política (FIOCRUZ, 2020). Esse estudo reflete o descrédito à ciência por parte da população brasileira, que tem negligenciado as medidas de segurança sugeridas por instituições globais de saúde e, assim, colocando vidas em risco. Dessa forma, evidencia-se a importância de projetos como este, que busca tornar o conhecimento científico acessível à comunidade acadêmica e à população geral.

No dia 15 de Fevereiro de 2020, o Dr. Tedros Adhanom Ghebreyesus, diretor geral da Organização Mundial da Saúde (OMS), disse, em uma coletiva de imprensa, que não estamos enfrentando apenas uma pandemia, mas sim uma "infodemia" (infodemic). Conforme a própria OMS, uma "infodemia" caracteriza a disseminação de muitas informações, incluindo informações falsas, em ambientes físicos e digitais, durante a emergência de uma doença (OMS, 2020). Isso significa que um dos principais desafios dos profissionais de saúde frente à pandemia da COVID-19 é gerenciar um gigantesco fluxo de informações, muitas vezes não confiáveis. Isso pode dificultar o enfrentamento 
à pandemia e a tomada de decisões informadas de maneira rápida (EL-JARDALI; BOUKARROUM; FADLALLAH, 2020).

Ao desenvolver tais atividades dentro do projeto, os discentes tiveram a oportunidade de entrar em contato direto com artigos científicos publicados em língua estrangeira, que traziam linguajar médico-científico. Ainda inexiste treinamento específico previsto na carga horária do curso de Medicina para utilização de programas de tradução ou edição de texto, tendo sido essa uma dificuldade previsível. De modo geral, os acadêmicos do Ensino Superior possuem apenas um nível intermediário de leitura/compreensão de textos científicos em seu idioma materno (CABRAL; TAVARES, 2005). Para tanto, é possível inferir que as dificuldades se intensificam quando esses textos estão, por exemplo, em língua inglesa, como é o caso dos artigos do projeto em questão. Além disso, ao extrapolar as generalizações, o aluno brasileiro não tem por hábito incluir a leitura como entretenimento ou ainda como meio de aquisição de conteúdo e crescimento pessoal. $\mathrm{O}$ que se observa é que, em sua maioria, as leituras se restringem àquelas obrigatórias do curso (TOURINHO, 2011). O projeto influenciou, portanto, na mudança desse padrão, uma vez que o ingresso por parte dos acadêmicos era voluntário, possivelmente selecionando um grupo mais interessado em leitura. Além de maior domínio na leitura de artigos científicos publicados na língua inglesa, os discentes também reforçaram o conhecimento teórico-prático sobre o novo vírus e a doença por ele causada. Conhecimentos no campo da Epidemiologia, Radiologia, Pediatria, Pneumologia, Clínica Médica, Ginecologia e Obstetrícia foram relatados como parte do desenvolvimento profissional dos acadêmicos.

Do ponto de vista dos docentes, a maior dificuldade inerente ao projeto foi distinguir as informações que seriam de fato relevantes para a comunidade. Apenas até abril de 2020, foram publicados mais de 1.239 artigos científicos sobre a COVID-19 (HAGHANI; BLIEMER; GOERLANDT; LI, 2020). O site "Retraction Watch" compila artigos que foram publicados e, posteriormente, retratados. Até o momento, 33 artigos sobre o novo coronavírus foram retratados, incluindo um artigo sobre os possíveis malefícios do uso da hidroxicloroquina, publicado após revisão por pares no The Lancet, jornal de renome, com alto fator de impacto (MEHRA; RUSCHITZKA; PATEL, 2020). Assim, apesar das qualificações técnicas e acadêmicas dos docentes, frente à constante oferta de publicações, foi laborioso selecionar as informações mais pertinentes para a área da saúde. 


\section{CONCLUSÃO}

A pandemia da COVID-19 envolveu o mundo todo e trouxe desafios inéditos, inclusive, à educação médica e à extensão universitária. Com o intuito de atender às necessidades sociais, optamos por desenvolver uma ação com caráter extensionista para disseminar o conhecimento científico sobre a COVID-19 para a comunidade. Dentro do contexto da educação em Ensino Superior, o projeto intitulado "Tradução livre de artigos científicos sobre a COVID-19" tornou-se uma estratégia de manutenção das atividades de extensão, mesmo com as limitações impostas pelo ensino à distância e isolamento social.

É essencial considerar que um sistema científico desenvolvido e uma cidadania informada requerem acesso público e imediato à pesquisa. Desta maneira foi possível fornecer informação científica atual e de qualidade à sociedade, com foco nos profissionais da área da saúde. Ao mesmo tempo, colaboramos para minimizar o impacto das fake news, possibilitando o enfrentamento da pandemia com maior segurança. $\mathrm{O}$ impacto secundário do projeto foi refletido na aquisição de conhecimento e desenvolvimento de novas habilidades aos acadêmicos de medicina nele envolvidos. De maneira geral, tanto docentes quanto discentes, julgaram toda a experiência de participação como muito positiva para a formação profissional.

\section{AGRADECIMENTOS}

Agradecemos o empenho de todos os alunos que participaram deste projeto, nomeadamente: Alessandra Antoniolli, Allan Leon Golfeto Pereira, André Jardim Domingues, Brenda Malucelli Rocha, Christyan Hydeaki Tamura Takahashi, Eluan Joel Rodrigues da Silva, Erika Yumi Takahashi, Flávia Renata Ropelatto Pires, Gabriela Rezende Vieira, Giovanna Dias Escarante, João Carlos Pegoraro de Almeida, João Lucas Lasta, João Vitor Taffarel, Lara Frangiotto Lopes, Larissa Panatta, Luan Gabriel Pinto, Lucas Augusto Marcon, Luís Fernando Rafalski Pereira, Manoela Ferreira da Cruz Neta, Marcela Dellatorre Pucci, Marcelo de Oliveira Machado, Mateus Henrique Verplotz, 
Natalie Toki Komori, Paola Bernardi da Silva, Rachel Yukie Toyama, Rodrigo Ribas Azzolini, Sofia Mitsue Ishie e Thiago de Carvalho Iocohama. Agradecemos também ao Leandro Felix de Santana, técnico administrativo da UFPR Campus Toledo, que auxiliou nas postagens para divulgação do projeto em redes sociais.

\section{REFERÊNCIAS}

ATALlAH, Álvaro Nagib. Medicina baseada em evidências. São Paulo Medical Journal, São Paulo, v. 136, n. 2, p. 43-44, mar. 2018. Disponível em: https://docs.bvsalud.org/biblioref/2018/06/904888/rdt_v23n2_43-44.pdf. Acesso em: 13 abr. 2021.

BARBOSA, DAVID SOEIRO. Saberes e Práticas da Extensão Universitária na Resposta ao Enfrentamento da COVID-19 no Brasil. Revista Práticas Em Extensão, v. 4, n. 1, p. 50-51, 2020.

BRASIL. MINISTÉRIO DA EDUCAÇÃO. RESOLUÇÃO $\mathbf{N}^{\mathbf{0}}$ 7, DE 18 DE DEZEMBRO DE 2018. 2018. Disponível em: http://portal.mec.gov.br/index.php?option=com_docman\&view=download\&alias $=1042$ 51-rces007-18\&category_slug=dezembro-2018-pdf\&Itemid=30192. Acesso em: 13 abr. 2021.

BRITTO, P. H. Tradução e ilusão. Estudos Avançados, [S. l.], v. 26, n. 76, p. 21 27, 2012. Disponível em: https://www.revistas.usp.br/eav/article/view/47535. Acesso em: 04 abr. 2021.

CENTRO ACADÊMICO DE MEDICINA DE TOLEDO (Toledo). Universidade Federal do Paraná. [Highlights] Infecção por SARS-CoV-2 em Crianças. Toledo, 25 mar. 2020. Instagram: @ca.medicinatoledo. Disponível em: https://www.instagram.com/p/B-LSptaFS6u/?igshid=1md0grtgiexd8. Acesso em: 13 abr. 2021.

DINIZ, Emily Gabriele Marques; SILVA, Adriana Maria da; NUNES, Paulo Henrique Valença; FRANCA, Wilza Wanessa Melo; ROCHA, João Victor Ritinto da; SILVA, Débora Verônica Sarmento Pereira da; SANTOS, Victor Hugo Barbosa dos; 
ARAÚJO, Hallysson Douglas Andrade de; ALBUQUERQUE, Mônica Camelo Pessoa de Azevedo; AIRES, André de Lima. A extensão universitária frente ao isolamento social imposto pela COVID-19. Brazilian Journal Of Development, [S.L.], v. 6, n. 9, p. 72999-73010, 2020. Brazilian Journal of Development. http://dx.doi.org/10.34117/bjdv6n9-669.

EL-JARDALI, Fadi; BOU-KARROUM, Lama; FADLALLAH, Racha. Amplifying the role of knowledge translation platforms in the COVID-19 pandemic response. Health Research Policy And Systems, [S.L.], v. 18, n. 1, p. 1-7, 3 jun. 2020. Springer Science and Business Media LLC. http://dx.doi.org/10.1186/s12961-02000576-y.

FUNAI, Anderson; SILVA, Tatiana Gaffuri da; ZANETTINI, Angélica; HANAUER, Marceli Cleunice; SILVA, Eduarda Luiza Maciel da; VALCARENGHI, Eduarda; CANCI, Julia; KLAUS, Georgia Baldo. O Processo de formação profissional frente à pandemia da Covid-19 / The vocational training process in the face of the Covid19 pandemic. Brazilian Journal Of Health Review, [S.L.], v. 3, n. 6, p. 19342-19348, 2020. Brazilian Journal of Health Review. http://dx.doi.org/10.34119/bjhrv3n6-314.

IOANNIDIS, John P. A. Coronavirus disease 2019: the harms of exaggerated information and non :evidence :based measures. European Journal Of Clinical Investigation, [S.L.], v. 50, n. 4, p. 1-5, abr. 2020. Disponível em: https://onlinelibrary.wiley.com/doi/full/10.1111/eci.13222. Acesso em: 06 abr. 2021.

LARIVIÈRE, Vincent; SHU, Fei; SUGIMOTO, Cassidy. The Coronavirus (COVID-19) outbreak highlights serious deficiencies in scholarly communication. 2020. Disponível em: https://blogs.lse.ac.uk/impactofsocialsciences/2020/03/05/thecoronavirus-covid-19-outbreak-highlights-serious-deficiencies-in-scholarlycommunication/. Acesso em: 13 abr. 2021.

LOPES, Plínio. Traduzindo a pandemia. 2020. Disponível em: https://piaui.folha.uol.com.br/traduzindo-a-pandemia/. Acesso em: 13 abr. 2021.

MEHRA, Mandeep R; RUSCHITZKA, Frank; PATEL, Amit N. RetractionHydroxychloroquine or chloroquine with or without a macrolide for treatment of COVID- 
19: a multinational registry analysis. The Lancet, [S.L.], v. 395, n. 10240, p. 1820, jun. 2020. Elsevier BV. http://dx.doi.org/10.1016/s0140-6736(20)31324-6 (retratado).

MENEGHINI, Rogerio; PACKER, Abel L. Is there science beyond English? Embo Reports, [S.L.], $\quad$ v. $\quad 8, \quad$ n. $2, \quad$ p. 112-116, fev. 2007. EMBO. http://dx.doi.org/10.1038/sj.embor.7400906.

NUNES, Ana Lucia de Paula Ferreira; SILVA, Maria Batista da Cruz. A extensão universitáriano ensino superiore a sociedade. Mal-Estar e Sociedade. Barbacena, p. 119133. 2011. Disponível em: https://revista.uemg.br/index.php/gticmalestar/article/view/60/89. Acesso em: 06 abr. 2021.

OLIVEIRA, Claudio Luiz. A importância da tradução: reflexões sobre o papel do tradutor. Communitas, Rio Branco, v. 1, n. 1, p. 351-356, Jan 2017. Disponível em: https://periodicos.ufac.br/index.php/COMMUNITAS/article/download/1109/pdf/. Acesso em: 13 abr. 2021.

ORGANIZAÇÃO MUNDIAL DA SAÚDE. Infodemic. Disponível em: https://www.who.int/health-topics/infodemic\#tab=tab_1. Acesso em: 13 abr. 2021.

ORGANIZAÇÃO PAN-AMERICANA DE SAÚDE. Entenda a infodemia e a desinformação na luta contra a COVID-19. 2020. Disponível em: https://iris.paho.org/bitstream/handle/10665.2/52054/FactsheetInfodemic_por.pdf?sequence=14. Acesso em: 13 abr. 2021.

PRADHAN P, Pandey AK et al. Uncanny similarity of unique inserts in the 2019nCoV spike protein to HIV-1 gp120 and Gag. BioRxiv. https://doi.org/10.1101/2020.01.30.927871 (retratado).

PRÓ-REITORIA DE EXTENSÃO E CULTURA - UNIVERSIDADE FEDERAL DO PARANÁ (Paraná). COEX - Modalidades de Extensão. 2021. Disponível em: http://www.proec.ufpr.br/links/extensao/modalidades_extensao.html. Acesso em: 13 abr. 2021.

REINO UNIDO. BRITISH COUNCIL. Learning English in Brazil: understanding the aims and expectations of the brazilian emerging middle classes. 2014. 
https://www.britishcouncil.org.br/sites/default/files/learning_english_in_brazil.pdf.

Acesso em: 13 abr. 2021.

DE BRITO E SILVA, Ana Lucia et al. Importância da Extensão Universitária na Formação profissional: Projeto Canudos. Revista de Enfermagem UFPR on line, [S.1], v. 13, out 2019. ISSN 1981-863. Disponível em < https://periodicos.ufpe.br/revistas/revistaenfermagem/article/view/242189> Acesso em 05 abr. 2021. Doi: httpps://doi.org/10.5205/1981-8963.2019.242189.

SERRÃO, ACP. Em Tempos de Exceção como Fazer Extensão? Reflexões sobre a Prática da Extensão Universitária no Combate à COVID-19. Revista Práticas em Extensão, v. 04, nº 01, 47-49, 2020.

UNIVERSIDADE FEDERAL DO PARANÁ (Paraná). CIÊNCIA ACESSÍVEL: grupo de alunos da ufpr se reúne para traduzir artigos científicos relacionados ao coronavírus. 21 maio 2020. Facebook: @UFPRoficial. Disponível em: https://www.facebook.com/UFPRoficial/posts/3890247737715379. Acesso em: 13 abr. 2021.

UNIVERSIDADE FEDERAL DO PARANÁ - CAMPUS TOLEDO (Paraná). Tradução livre de artigos científicos sobre o COVID-19. 2020. Disponível em: http://www.toledo.ufpr.br/portal/wpcontent/uploads/2020/03/Infec\%C3\%A7\%C3\%A3o-por-SARS-CoV-2-emCrian\%C3\%A7as.pdf. Acesso em: 13 abr. 2021.

Recebido em: 16 de abril de 2021. Aceito em: 20 de abril de 2021. 